\title{
Miracurall: The Electronic Cure for Diabetes Mellitus
}

\author{
Abhijit Naskar \\ Inventor, Independent Research Scientist, Kolkata, India
}

\begin{abstract}
The entire galaxy of ours is a giant collection of vibrating strings. Starting from the tiniest molecular level of all materials till the unimaginable vast multiverses - everywhere we can see resonance, not with the eyes under the frontal lobe but with the eyes of M-theory. Harmony is a natural instinct of the cosmos. Every tiny particle that exists in the material world and beyond, has its own natural resonance in which it is constantly vibrating, thus maintaining harmony in the universe (in human conceivable term). When it comes to molecular biology, we call it "homeostasis". Anatomy of all living beings is also a collective form of vibrating molecules in their natural resonance. Thus, once human mind is focused on the tiniest region of human body, there we can discover homeostasis in a resonating form. The body is working according to human will, only because at the molecular level each particle is vibrating at its own pace. And whenever, the dreadful warfare of modern lifestyle attacks this beautiful harmony of the most amazing creation of mother nature, the natural rhythm of human anatomy gets wrecked. As a very easy consequence of it, the organs that keep the body running in its daily needs, are forced to malfunction. Thus, another disgraced yet inevitable term emerges, i.e. "chaos". Quite fortunately, as this version of chaos is trapped inside human body, it can be replaced with the lost harmony or in this case "homeostasis". The way is to make the mal-functional particles at the molecular level vibrate in its natural rhythm again, for which a triggering energy is provided inside the body artificially. It reinitiates the homeostasis mechanism in the necessary organs. And, the little electronic device that creates this triggering energy, is called "MIRACURALL".
\end{abstract}

\section{Introduction}

Human body is a complex system of hardware. The organs inside are different components of this system. Like a modern day computer, this beautiful hardware needs software programs to run according to human will. The Central Nervous System (CNS) drives the entire system with the use of neural pulses. Once the human hardware components start to malfunction, a copy of the same software program has to be reinstalled for the specific organ. Miracurall forms the triggering energy called the "Miracurall Pulses (M-Pulses)" as a mostly alike copy of the rudimentary software program. M-Pulses reinstate the refreshed beginning of the malfunctional components or in this case "organs" of the human body. With a proper software program each organ or region of human body can be repaired or restored to start working again normally. Thus M-Pulses make "homeostasis" prevail inside human anatomy starting the limitless journey from the pancreatic hormone regulation system.

\section{The Rhythmic Nature of Human Body and The Amazing Effect of External Energy Pulsation on the Human Anatomy}

At all levels, nature is a composite of rhythms. Vibrations underlie virtually every aspect of nature. The vibrations of atoms create sound and heat. Light arises from the vibrations of electrons in an object. When we say something is blue, what is really happening is that light has made the electrons within the object vibrate in a way that causes the emission of blue light. At a basic level, all life depends upon molecules interacting through vibrating or oscillating energy fields. Virtually all that we know about living systems and even to a deeper sense non-living system as well, based on the analysis of vibrations. Life is immersed in this spectrum and contributes its own unique set of rhythms. In the living body, each electron, atom, chemical bond, molecule, cell, tissue, organ (and the body as a whole) has its own vibratory character. Since living structure and function are orderly, biological oscillations are organized in meaningful ways, and they contribute information to a dynamic vibratory network that extends throughout the body and into the space around it. Superimposed upon that rhythm, are many cycles of replacement of the atoms comprising the body (Schoenheimer 1942). Some tissues, such as bone and fascia, are completely replaced some 10-15 times during a lifetime, while others, such as skin and intestine, are replaced 10000 times during the same period. Certain enzymes last only few seconds before they are renewed (Ratner 1979). Each organ has its own set of activity rhythms, such as the ovary, with its monthly cycle. Shorter yet are the rhythms of the cranio/sacral pulse, the breath, the heartbeat and most importantly the brain waves. Even shorter are the vibrations of molecules, which spin, wiggle and shake millions of times each second.

Now, let's focus a bit of attention to the rhythmic functioning of human brain - perhaps so far the most delicate and inexplicable gift of the cosmos. The EEG (Electroenecephalograph) measures the brain waves of 
different frequencies within the brain. Raw EEG frequency bands include gamma (25-60 Hz); beta (12-25 Hz); alpha $(7-12 \mathrm{~Hz})$; theta $(4-7 \mathrm{~Hz})$; and delta (below $4 \mathrm{~Hz}$ ). These frequencies are linked to behaviors, subjective feeling states, physiological correlates, etc. Brain waves are not constant in frequency, but vary from moment to moment. The "pacemaker" or "rhythm section" is located deep in the brain, specifically in the thalamus. The system is known as the thalamic rhythm generator or pacemaker (Anderson \& Anderson 1968). Careful research is determining the cellular basis of the rhythms (Destexhe et al 1993, Wallenstein 1994). Calcium ions slowly leak into single thalamocortical neurons, which oscillate for 1.5-28 seconds, triggering and entraining the brain waves, which spread upward throughout the brain. Eventually the thalamic oscillations cease because of the excess calcium built up in the thalamocortical neurons. During the 'silent phase', lasting from 5 to 25 seconds, the brain waves are susceptible to entrainment by external fields. Eventually the thalamic oscillations begin again, after the cells have restored their calcium levels in the point where they are once again able to oscillate. The EEG waves spread not only throughout the brain, but throughout the entire nervous system (especially via the mysterious wanderer nerve named as "vagus") and into part of the organism. In this way the brain waves regulate the overall sensitivity and activity of the entire nervous system.

Now with the amazing human consciousness, let's dig deeper to discover the eloquence of the rudimentary link between the human nervous system and geophysical rhythm of our beloved planet earth. Lewis B. Hainsworth of Western Australia seems to be the first researcher to recognize the relationship of brain wave frequencies to the naturally circulating rhythmic signals, known as Schumann's Resonance (SR). In 1952, a German physicist, Dr W.O. Schumann, suggested that the space between the surface of the earth and the ionosphere should act as a resonant cavity, somewhat like the chamber in a musical instrument. Pressing the keys on a wind instrument changes the size of the cavity and therefore changes the frequency of the standing waves within that cavity. Energy for the SR is provided by lightning. Lightning pumps energy into the earthionosphere cavity and causes it to resonate at frequencies in the ELF range. In 1957, Dr Schumann calculated the SR frequencies and fixed the most predominant standing wave at about $7.83 \mathrm{~Hz}$. Following the cue from Schumann, Hainsworth, was among the first to suggest that human health is linked with geophysical parameters by way of the naturally occurring Schumann's ELF. His hypothesis identified naturally occurring features which determine the frequency spectrum of human brain wave rhythm:

The frequencies of naturally occurring electromagnetic signals, circulating in the electrically resonant cavity bounded by the Earth and the ionosphere, have governed or determined the 'evolution' or development of the frequencies of operation of the principal human brain wave signals. In particular, the alpha rhythm is so placed that it can in no circumstances suffer an extensive interference from naturally occurring signals.

Hainsworth concluded that the frequencies of human brain waves evolved in response to these signals. If this hypothesis is correct, conditions for evolutionary changes in human brain wave patterns have now been established. Furthermore, variations in these patterns can produce mild to disastrous health and behavioral changes. A tuned system consists of at least two oscillators of identical resonant frequencies. If one oscillator starts emitting, the other will be activated by the signal very shortly, in the process of resonance, entrainment or kindling (igniting the resonance phenomenon among the neurons). It becomes obvious that in deep meditation, when waves of alpha and theta rhythms cascade across the entire brain, a resonance is possible between the human being and the planet. Energy and information which are embedded in a field are transferred. Perhaps the planet communicates with man in this primal language of frequencies. The frequencies of SR signals change with ionospheric conditions. These conditions change diurnally, seasonally and with variations in solar activity, which, in turn varies with the 11 year sunspot cycle and also with the 27-29 day lunar cycle, mainly during sunspot minimum periods. Lunar tidal changes in the height and thickness of the layers could also sometimes affect the cavity dimensions and hence the Schumann's frequencies. Hence, these changes in the atmospheric conditions sometimes affect the biological systems. For instance, lunar cycle creates ionospheric changes, which in turn proves to be the precursor and stimulant to rheumatoid arthritic pain in some aged people. A number of biologists have concluded that the frequency overlap of SR and biological fields is not accidental, but is the culmination of a close interplay between geomagnetic and biomagnetic fields over evolutionary time (e.g. Direnfeld 1983). Hence researchers have examined interactions between external fields and biological rhythms. Organisms are capable of sensing the intensity, polarity and direction of the geomagnetic field (Gould 1984). A variety of behavioral disturbances in the human population are statistically related to disturbances in the earth's electromagnetic field or to man-made interferences :

* Friedman et al (1965) documented a relationship between increased geomagnetic activity and the rate of admission of patients to 35 psychiatric facilities.

* Venkatraman (1976) and Rajaram \& Mitra (1981) reported an association between changes in the geomagnetic field due to magnetic storms and frequency of seizures in epileptic patients.

* Perry et al (1981) correlated suicide locations in the West Midlands, England, with high magnetic field strengths due to $50 \mathrm{~Hz}$ power lines. 
Many studies have demonstrated the probable entrainment of brain waves by external rhythms of natural and artificial origin :

* Reiter (1953) measured reaction time, an important factor in traffic safety. Upon entering a cubicle at a traffic exhibition, visitors were asked to press a key. When a light came on, they were to release pressure on the key. Their reaction time (i.e. the time between 'light on' and 'key release') was recorded for many thousands of visitors over a 2 month period. At the same time, the ELF micropulsations (SR) were monitored. The micropulsations slow down when a thunderstorm is approaching, and Reiter found that the subjects were slower to respond during such periods. When the micropulsations speeded up, into the range of alpha brain wave activity, reaction times were faster.

* After the traffic exhibition, Reiter took his test cubicle in the University of Munich and lined the top to bottom with wire mesh connected to an electrical generator. He introduced artificial low level, low frequency signals similar to those of the earth's field. Under these controlled conditions, the effects of the fields on reaction time were comparable to those obtained during the exhibition. Moreover, subjects in the laboratory experiments repeatedly complained about headaches, stiffness in the chest, and sweating of the palms after several minutes of exposure to 3 cycles/second fields. When the headaches faded away, there was often a feeling of fatigue. These symptoms resemble the so-called 'weather sensitivity' complaints that some people have before the arrival of thunderstorm.

* Over many years, Wever (1968) and colleagues at Max Planck Institute in Germany observed hundreds of subjects who lived in two underground rooms that were shielded from external rhythms of light, temperature, sound, pressure etc. One room also had an electromagnetic shield around it, consisting of a mesh of steel rods and plates that reduced the influence of geomagnetic rhythms by $99 \%$. The rhythms of body temperature, sleep-waking, urinary excretion and other physiological activities were monitored. All subjects developed longer and irregular or desynchronized or chaotic physiological rhythms. Those in the magnetically shielded room developed significantly longer and more irregular physiological rhythms. In some experiments, artificial electric and magnetic rhythms were pushed into the shielding. Only one field had any effect : a very weak $10 \mathrm{~Hz}$ electric field. This field dramatically restored normal patterns to biorhythm measurements.

Each of these important but seldom cited studies concluded that biological rhythms can be entrained with natural and artificial ELF electric fields. Entrainment of brain waves can set the overall speed of responsiveness of the nervous system to stimulation. Likewise, with externally provided pulsating energy fields, every organ of human anatomy can be triggered to vibrate again in a healthy manner, so that homeostasis is reinstated inside the body. A variety of electrical, electronic, magnetic and other energetic phenomena take place within healthy tissue as a consequence of the communications needed to coordinate cellular activities and restore homeostasis. Whether caused by physical or emotional trauma, the wound that does not heal, is a wound that is not receiving the natural regulatory signals needed to initiate and coordinate repair process. Hence, certain accurately administered triggering energy pulsations in the form of "M-Pulses" trigger the regeneration of damaged and diseased cells, re-harmonizes the human biological system using the phenomenon of entrainment.

\section{Diabetes Mellitus in Brief}

So, as the rhythmic nature of human organisms and its quite evident link with external energy pulsation are established, let's embark on the prosperous journey of countless cures, beginning with Hyperglycemia or in layman's term Diabetes. 'Hyperglycemia' originates from the Greek word parts hyper (too much), glyco (sugar) and emia (condition of the blood). Diabetes Mellitus (DM), as you all know, is a chronic metabolic disorder, which results either from failure of the pancreas to produce insulin or from insulin resistance, with inadequate insulin secretion to sustain normal metabolism. DM damages blood vessels, nerves, kidneys, the retina and in pregnancy, the developing fetus and the placenta. DM is currently established if patients have classic diabetic symptoms and if on two occasions fasting glucose levels exceed $126 \mathrm{mg} / \mathrm{dl}(7 \mathrm{mmol} / \mathrm{L})$, random glucose levels exceed $200 \mathrm{mg} / \mathrm{dl}(11.1 \mathrm{mmol} / \mathrm{L})$, or a 2 -hour oral glucose tolerance test is $200 \mathrm{mg} / \mathrm{dl}$ or more. Type-1 DM is caused by the autoimmune destruction of the insulin producing beta $(\beta)$ cells of the pancreas. The loss of these cells results in nearly complete insulin-deficiency. Type-1 DM (formerly called 'juvenile-onset' DM) occurs usually in children, although extremely obese children are now being diagnosed with Type-2 DM as well. Type2 DM results partly from a decreased sensitivity of muscle cells to insulin-mediated glucose uptake and partly from a relative decrease in pancreatic insulin secretion. Type-2 DM primarily affects obese middle-aged people with sedentary lifestyle. 
Miracurall: The Electronic Cure For Diabetes Mellitus

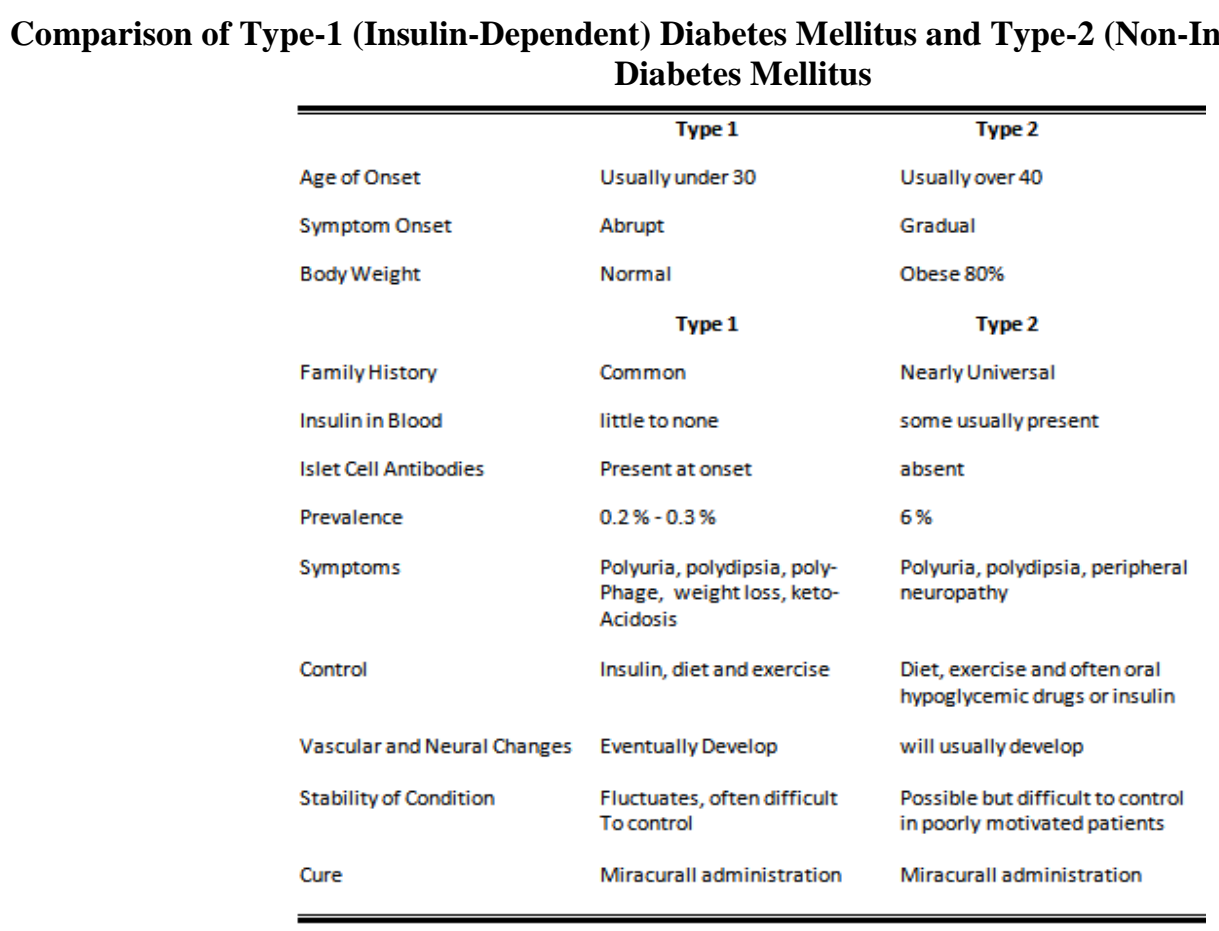

Without insulin, cells remain relatively impermeable to glucose and cannot obtain enough from the blood. The individual experiences fatigue as the cells become starved for glucose. The body compensates to some degree by switching to protein and fat metabolism for energy. Fats and proteins are less accessible, however, and more difficult than glucose to break down. Fat metabolism also releases ketones, such as acetone, as a toxic by-product, which can be smelled on the breath. The kidney is very efficient under normal conditions; however, at plasma glucose concentrations above about $180 \mathrm{mg} / \mathrm{dl}$, the kidney pump becomes saturated. Glucose from concentrations above $180 \mathrm{mg} / \mathrm{dl}$ therefore ends up in the urine. This changes the osmotic gradient across the nephrons of the kidneys. Large volumes of water therefore follow the glucose by osmosis into the kidneys, and get excreted. People with untreated diabetes experience low energy and great thirst, and produce large volumes of glucose-rich urine. In the long-term, continued high levels of blood glucose can lead to blindness, kidney failure, nerve damage, and gangrene (a severe infection) in the limbs.

In this context, the organ that might feel most insulted, if I don't talk about it, is the pancreas. Pancreas has the most important and delicate role in monitoring blood-glucose level. The pancreas has two major functions: it produces and releases digestive enzymes, and two major hormones responsible for the endocrine control of glucose metabolism: insulin and glucagon. The exocrine pancreas comprises about $98 \%$ of the mass of the organ. The acinar cells synthesize and secrete the digestive enzymes (trypsin, chymotrypsin, elastase, amylase, and others) that break down food into simpler components that can be absorbed by the intestine. The endocrine pancreas is comprised of small groups of cells distributed throughout the organ. These groups, the Islets of Langerhans, consist of four cell types $(\alpha, \beta, \delta$ and $F)$. The islet composition varies in different parts of the pancreas (posterior primarily F-cells with some $\beta$-cells, and anterior primarily $\beta$-cells with some $\alpha$ and smaller amounts of $\delta$-cells). Each cell type secretes one major hormone: the $\beta$-cells are the insulin producing cells, while the $\alpha$-cells produce glucagon, the $\delta$-cells produce somatostatin, and the F cells produce Pancreatic Polypeptide. Insulin is released from the $\beta$-cells in response to elevated plasma glucose, mannose, and some amino acids, especially leucine. Stimulation of insulin release by glucose can be enhanced by other hormones (especially those released by the gut, such as gastrin inhibitory peptide and cholecystokinin; this is why insulin release due to oral administration is greater than release due to intravenous infusion of glucose), by arginine and some other amino acids, and by $\beta$-adrenergic agonists. Insulin release is inhibited by somatostatin, by cortisol, and by catecholamines acting via $\alpha$-adrenergic receptors. Although specific $\alpha$ and $\beta$-adrenergic agonists have opposite effects on insulin release, the net effect of physiological catecholamine action is strongly inhibitory. Prolonged high levels of glucose decrease the $\beta$-cell response to the glucose stimulation, without altering $\beta$-cell responsiveness to other stimuli. Although not completely understood, this appears to be due at least in part to a decrease in the amount of GLUT2 glucose transporter in the $\beta$-cell membrane. Prolonged elevation of glucose (such as that caused by hypersecretion of glucocorticoids) may exhaust the $\beta$-cell stores of insulin and exceed the ability of the $\beta$-cell to synthesize additional hormone, resulting in hyperglycemia; it is possible that this down-regulation of GLUT2 is one mechanism for reducing risk of $\beta$-cell exhaustion. Transcription of the insulin 
gene and translation of the insulin mRNA are stimulated by glucose. Acute increases in glucose levels largely affect secretion of pre-formed insulin from secretory vesicles and mRNA translation and stability, while chronic increases in glucose levels increase transcription of insulin mRNA. Increases in transcription and translation require some time to become effective. Stimulation with glucose may therefore result in two phases of insulin release; a rapid phase, due to release of pre-formed hormone from mature vesicles, and a slower phase requiring synthesis of new protein. During onset of Type $1 \mathrm{DM}$ the rapid phase disappears first, because basal insulin secretion rates become such a large proportion of the remaining $\beta$-cell capacity that storage of pre-formed insulin in vesicles is no longer possible.

Insulin action is mediated by the insulin receptor, a complex multi-subunit cell surface glycoprotein. Binding of insulin activates the intrinsic tyrosine kinase activity of the receptor. Tyrosine phosphorylation of the insulin receptor itself and of some other proteins is thought to be required for insulin action. As a result of these phosphorylation (and probably other) events, a number of other intracellular proteins are activated, including several kinases, phosphatases, and transcription factors. One important enzyme activated by the second messenger cascade is phosphodiesterase, which decreases cellular cAMP levels. Acute increases in insulin levels result in phosphorylation or de-phosphorylation of existing proteins (e.g. the enzymes involved in the glycolytic and gluconeogenic pathways), which have rapid but transient effects on their activity. High levels of insulin, or prolonged stimulation by insulin results in alterations in the rate of transcription of a variety of genes, and therefore in increased or decreased capacity for the processes mediated by those gene products. Prolonged high levels of insulin result in desensitization (de-coupling of the receptor from the cellular responses) and down-regulation (decreased amounts of the receptor on the cell-surface). These phenomena protect the cell from overstimulation (similar effects are seen with a number of other hormones). Desensitization and downregulation of the insulin receptor are important in Type $2 \mathrm{DM}$, since these phenomena occur upon prolonged high levels of insulin and contribute to the insulin resistance that characterizes the disorder. It is therefore preferable, although not always possible, to avoid use of exogenous insulin for Type $2 \mathrm{DM}$, since this tends to further down-regulate the receptor and worsen the desensitization that has already occurred.

The liver is the main site of action of the pancreatic hormones. Because blood flow from the pancreas proceeds directly to the liver, and because the liver is the major site of inactivation of most peptide hormones, the liver is exposed to higher levels of pancreatic hormones than any other tissue. In liver, insulin stimulates glycogen synthesis and inhibits glycogen breakdown. It also stimulates glycolysis and inhibits gluconeogenesis. In addition to its effects on glucose metabolism, insulin has a variety of anabolic actions in the liver, stimulating lipid synthesis and release and protein synthesis, and inhibiting the breakdown of these compounds. The liver has some ability to respond directly to high levels of plasma glucose by increasing glucose uptake and glycogen synthesis in an insulin-independent manner; however, the majority of liver glucose regulatory functions require insulin action. The liver has about 300,000 insulin receptors per cell (a very large number), but experiences maximal response to insulin when a small fraction of the receptors are occupied; this allows the organ to respond to insulin even when the plasma insulin concentration is less than the Kd for the receptor. In muscle, insulin stimulates amino acid uptake and protein synthesis, and glucose uptake and incorporation into glycogen. The muscle plays an important role in absorbing the majority (80-95\%) of sudden increases in plasma glucose levels, such as those observed during a rich carbohydrate meal. The muscle expresses significant amounts of the GLUT4 glucose transporter, which, upon insulin stimulation, is translocated to the plasma membrane, allowing a massive increase in glucose uptake. During exercise the muscle becomes more sensitive to insulin action and therefore retains the ability to import glucose from circulation in spite of the exercise-induced reduction in insulin levels.

The other major regulatory hormone of the pancreas is glucagon, release of which from the $\alpha$-cells is stimulated by low plasma glucose and by catecholamines and glucocorticoids. Release of glucagon is inhibited by insulin and somatostatin. Release of glucagon is also inhibited by glucose; it is not known whether this is a direct effect of glucose on the $\alpha$-cell, or an indirect consequence of elevated insulin levels. Glucagon has actions opposite to those of insulin, and therefore functions to maintain plasma glucose levels between meals. However, unlike insulin, glucagon action is probably limited to the liver, with limited effects in other tissues. Glucagon stimulates liver amino acid uptake, gluconeogenesis, and glucose release, and inhibits glycolysis and fatty acid synthesis. The glucagon receptor is coupled to adenylyl cyclase, and glucagon actions are mediated by elevation in cAMP levels. In general, actions of glucagon are mediated by increased phosphorylation of existing enzymes. Prolonged stimulation by glucagon may have some effects on gene transcription, usually in the opposite direction from that of insulin. If the insulin:glucagon ratio is low for a prolonged period (i.e. several days), an alteration in liver enzyme levels occurs, which causes increased production of ketone bodies.

One important regulator of pancreatic hormone release is the paracrine signaling that occurs within the islets. Insulin inhibits its own release, and also inhibits the release of glucagon. Glucagon inhibits its own release, and stimulates insulin release. Somatostatin inhibits the release of both insulin and glucagon. The local changes in hormone levels act as a primary negative feedback mechanism to prevent excessive changes in 
pancreatic hormone levels. Hence, effects of untreated Type 1 DM could be utterly dangerous, in which the loss of insulin inhibition of glucagon secretion results in overproduction of glucagon; the high levels of glucagon in the absence of insulin action throughout the body result in the severe metabolic changes of diabetic ketoacidosis.

The pancreas alters its release of insulin and glucagon in response to changes in plasma glucose and other circulating nutrients. One major cause of these changes is eating. The response to a meal varies significantly depending on the composition of the food. Intravenous infusion of glucose elicits a smaller rise in insulin release than does oral administration of an equivalent amount of glucose. The greater increase in insulin levels caused by actually eating is thought to be due to gastrointestinal peptide hormones. These peptides are released in response to food absorption and potentiate the glucose effect on insulin release. When eating a meal rich in carbohydrate, insulin levels rise and glucagon levels fall. The decrease of glucagon is due to inhibition of its release by insulin, and to the elevation in plasma glucose. When eating a meal rich in protein, insulin levels rise, because insulin secretion is stimulated by amino acids. Glucagon levels also rise; glucagon release is also stimulated by amino acids. In this case, unopposed insulin action would result in hypoglycemia, since little glucose is being absorbed; glucagon must increase to maintain plasma glucose. When eating a mixed meal, insulin levels rise, and glucagon levels rise, fall, or remain unchanged as appropriate to maintain plasma glucose. The pancreas uses its ability to monitor the influx of nutrients, supplemented by signals in the form of intestinal peptide hormones, to regulate the disposal of the nutrients without allowing an undue change in plasma glucose. Mimicking this tailored change in pancreatic hormone release is difficult to achieve by injections of insulin, and explains part of the problem faced by individuals with Type 1 DM. That is why, it is necessary to make the pancreatic hormone regulation system healthy again for a natural regulation of blood glucose which can be done with M-Pulses.

\section{The Pathway of Curing Type 1 and Type 2 Diabetes Mellitus with Miracurall}

The endocrine pancreas is richly innervated with sympathetic and parasympathetic projections from the brain. In the mid-20th century, it was established that $\alpha$-adrenergic activation inhibits, whereas cholinergic stimulation promotes, insulin secretion; this demonstrated the importance of the sympathetic and parasympathetic systems in pancreatic endocrine function. It was later established that insulin injected peripherally could act within the brain, leading to the discovery of insulin and insulin receptors within the brain and the receptor mediated transport of insulin into the central nervous system from endothelial cells. The insulin receptor within the central nervous system is widely distributed, reflecting insulin's diverse range of actions, including acting as an adiposity signal to reduce food intake and increase energy expenditure, regulation of systemic glucose responses, altering sympathetic activity, and involvement in cognitive function. As observed with central insulin administration, the pancreatic hormones glucagon, somatostatin, pancreatic polypeptide, and amylin can each also reduce food intake. In this case, I was more interested in the parasympathetic innervations in the pancreas and liver, as M-Pulses make use of the vagally mediated parasympathetic rhythmic signals to start the halted rhythmic regeneration process of the islet cells in the pancreas and pancreatic hormone receptors in the regions like liver. In this context, evidence consistent with a functional role of the nervous system in the pancreatic islets was accumulating. Porte demonstrated that local $\alpha$-adrenergic stimulation inhibits secretion, whereas $\beta$-adrenergic agonists stimulate insulin secretion, strongly suggesting that insulin secretion is under sympathetic control. Campfield and colleagues subsequently observed that insulin secretion is stimulated by acetylcholine, indicating parasympathetic involvement. Cholinergic stimulation of insulin release was, in turn, decreased in the presence of epinephrine, implicating a complex neural control involving both parasympathetic and sympathetic control over $\beta$-cells. These early studies paved the way to the currently supported view that the autonomic nervous system can have a powerful influence over the pancreatic hormone regulation system. In the late 1960s and early 1970s, using a Pavlovian behavioral paradigm whereby an unconditioned stimulus is paired with a neutral stimulus over the course of several trials resulting in the neutral stimulus becoming a conditioned stimulus, S. C. Woods found that rats could be conditioned to secrete insulin and become hypoglycemic. In training sessions, experimental rats received a subcutaneous injection of insulin (the unconditioned stimulus) in the presence of a novel stimulus (usually an odor, the conditioned stimulus). Blood glucose decreased in response to insulin (the unconditioned response), whereas it increased slightly in control rats administered a placebo (saline) injection subcutaneously in association with the odor. After several such conditioning trials, a test day occurred in which all rats received only saline injections plus the odor, and those that previously received insulin became hypoglycemic. Subsequent experiments revealed that the conditioned hypoglycemia required an intact vagus nerve, could be blocked with the anti-cholinergic drug atropine, and was secondary to conditioned secretion of pancreatic insulin. Substantial new information has accumulated on the mechanisms of secretion, the development, and regulation of the gene expression, and the role of growth factors in the differentiation, growth, and regeneration of the pancreas. Many genes that are required for pancreas formation are active after birth and participate in endocrine and exocrine cell functions. Although the factors that normally regulate the proliferation of the pancreas largely remain elusive, several factors to influence the growth have 
been identified. It was also reported that the pancreas was sensitive to a number of apoptotic stimuli. The autonomic nervous system influences many of the functions of the body, including the pancreas and the liver as well. In fact, the parasympathetic nervous system and the sympathetic nervous system have opposing effects on insulin secretion from islet beta cells; feeding-induced parasympathetic neural activity to the pancreas stimulates insulin secretion, whereas stress-induced sympathetic neural activity to the pancreas inhibits insulin secretion. Moreover, it has been reported that the autonomic nervous system is one of the important factors that regulate pancreatic regeneration and stimulate the carcinogenesis. This implies that when proper support is provided to the Central Nervous System, it has the amazing ability to start halted regeneration process in the pancreas, liver and some other regions of the body as well

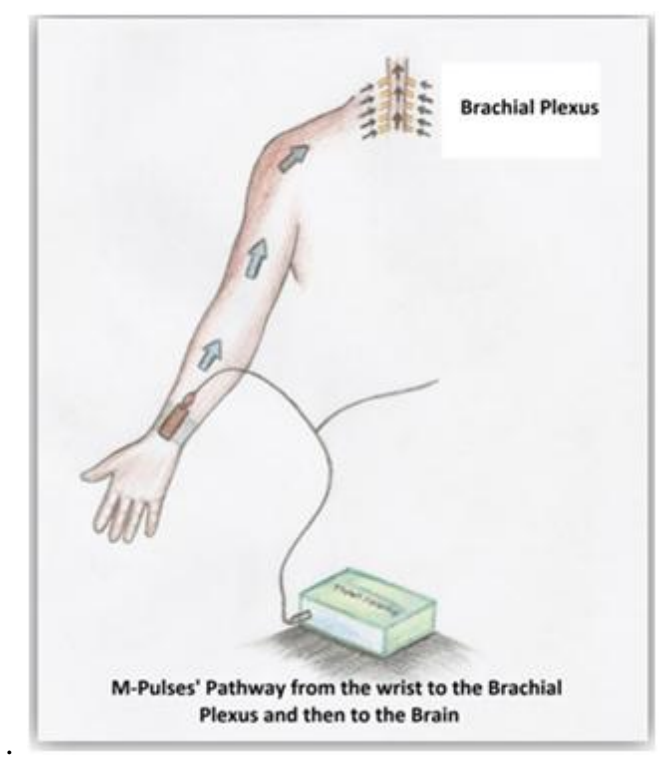

In the Miracurall administration, at first M-Pulses enter the body through the wrists and walking down the pathways of radial, musculocutaneous, ulnar and median nerves, they reach the beautiful brain. Now, let's engage the consciousness a little towards those pathways.

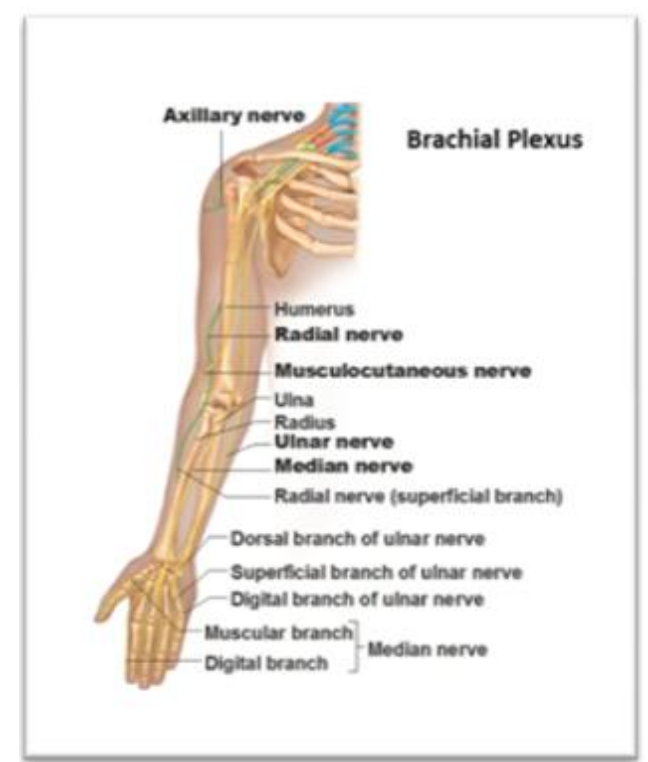

Radial Nerve : The radial nerve emerges from the posterior cord of the brachial plexus and descends within the deep aspect of the posterior arm. About midway down the shaft of the humerus, it lies against the bone in the radial groove. The radial nerve innervates all of the extensor muscles of the upper limb, the supinator muscle, and the brachioradialis. Its cutaneous sensory distribution is to the posterior portion of the upper limb, including the posterior surface of the hand. 
Musculocutaneous Nerve : The musculocutaneous nerve emerges from the lateral cord of the brachial plexus and provides motor innervation to the anterior muscles of the arm as well as cutaneous sensory innervation to part of the forearm.

Ulnar Nerve : The ulnar nerve emerges from the medial cord of the brachial plexus and innervates two forearm muscles plus most of the intrinsic hand muscles, except some associated with the thumb. Its sensory distribution is to the ulnar side of the hand.

Median Nerve : The median nerve emerges from the medial and lateral cords of the brachial plexus and innervates all but one of the flexor muscles of the forearm and most of the hand muscles at the base of the thumb, called the thenar area of the hand. Its cutaneous sensory distribution is to the radial portion of the palm of the hand.

The collective cutaneous sensory distribution of radial, musculocutaneous, ulnar and median nerves, acts as a gateway towards the Central Nervous System for Miracurall. M-Pulses enter the sensory region and through the pathways of those nerves they reach the brachial plexus of the spinal cord. The Brachial Plexus then sends the delicately formed rhythmic pulses directly to the brain and right away the brain transmits regeneration signal through the vagus nerve to the pancreas and liver for restarting the regeneration and healing process of the islet cells and pancreatic hormone receptors.

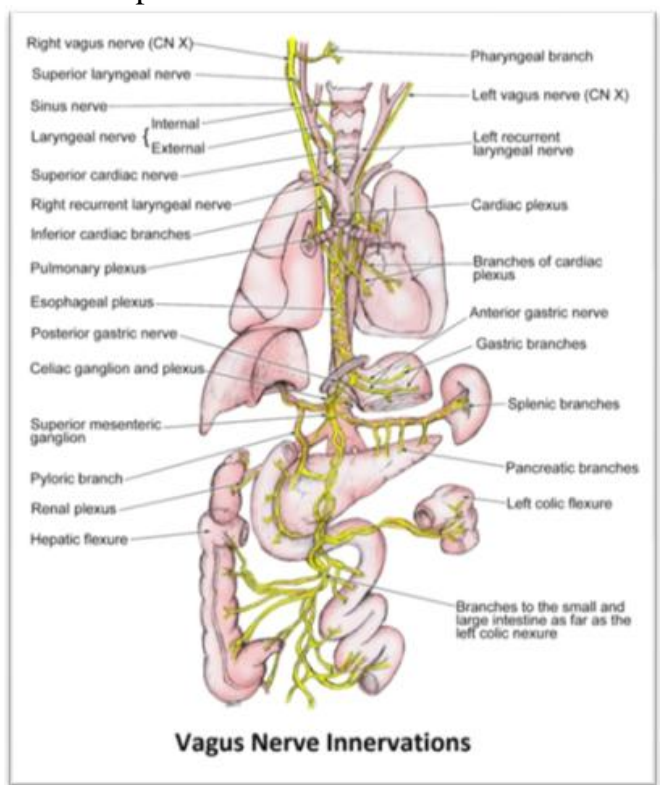

Vagus Nerve : The vagus nerve is a mixed cranial nerve that consists of $\sim 80 \%$ afferent fibres originating from the heart, aorta, lungs and gastrointestinal tract and $\sim 20 \%$ efferent fibres that provide parasympathetic innervations of these structures including pancreas and liver, and also innervate the voluntary striated muscles of the larynx and pharynx. Somata of the efferent fibres are located in the dorsal motor nucleus and nucleus ambiguus. Afferent fibres have their origin in the nodose ganglion and primarily project to the nucleus of the solitary tract. At the cervical level the vagus nerve mainly consists of small diameter unmyelinated C-fibres (65$80 \%$ ), with a smaller portion of intermediate-diameter myelinated B-fibres and large-diameter myelinated Afibres. The nucleus of the solitary tract has widespread projections to numerous areas in the forebrain, as well as the brainstem, including amygdala and thalamus. There are direct neural projections into the raphe nucleus, which is the major source of serotonergic neurons, and indirect projections to the locus coeruleus and A5 nuclei, which contain noradrenegic neurons. Finally, there are numerous diffuse cortical connections. Relatively few specific functions of the vagus nerve have been well characterised. The vagus nerve is often considered to be protective, defensive and relaxing. This primary function is exemplified by the lateral line system in fish, the early precedent of the autonomic nervous system. The control of homeostatic functions by the central nervous system in these earlier life forms was limited to the escape and avoidance of perturbing stimuli or suboptimal conditions. Its complex anatomical distribution has earned the vagus nerve its name, as 'vagus' is the Latin word for wanderer. In this context, some thoughts might raise in the air considering that, Miracurall is somewhat similar to Antiepileptic Vagus Nerve Stimulation. But in fact, Vagus Nerve Stimulation has no such connection with Miracurall, as this wanderer nerve is just our man to carry the parasympathetic rhythmic potential of regeneration to the pancreas and liver. Although, parasympathetic innervation is well established in many regions of the body, the accurate pulsating energy form of the M-Pulses, pose a healing effect mostly over the islet cells and the pancreatic hormone receptors in the liver. But even if M-Pulses trigger the regeneration process of the islet cells, one hiccup still remains, i.e. the auto-immune destruction of the $\beta$-cells. 
The somatosensory and autonomic nervous systems play an active role in host defense via direct regulation of immunological and inflammatory responses in the periphery. The auto-immune destruction of the $\beta$-cells in Type-1 DM is due to the defective and damaged $\beta$-cells. When the immune system notices defective cells in the pancreas, to protect the neighbouring cells and organs from further damage, it destroys those damaged $\beta$-cells, thus giving rise to Type-1 DM. But again when all the $\beta$-cells start working in a healthy manner, they no more pose a threat to the immune system, so the immune no more acts on the destruction of those cells. Hence, to put it in a nutshell, M-Pulses reinitiate the halted regeneration and healing process in the pancreas and the liver. Which means that homeostasis is prevailed in the pancreatic hormone regulation system. Hence, the hormonal regulation of the blood-glucose gets restored, giving rise to a completely cured state from Type-1 and Type-2 DM with no more need of medications and insulin injections.

\section{The Administration Procedure of Miracurall}

Now, I'll elaborate on the exact procedure of administering Miracurall on an individual. From a outward view, the main processing unit of Miracurall looks like a plain box with several control units and indicators on it. This Processing Unit forms nine different rhythms of electronic pulses. These rhythmic direct current mild electronic pulses, when administered on Hyperglycemic individuals in a certain time pattern, reinitiate the regeneration and healing process of the pancreatic hormone regulation system. In the front side of the Processing Unit, there are : switch for changing rhythm forming stage between A, B and C, a knob for controlling the amplitude of the signal in order to adjust the comfort of the individual, a rotary switch for changing rhythms of the stage $\mathrm{C}$ which contains seven different frequencies $(\mathrm{C} 1-\mathrm{C} 7)$. I'm not revealing the frequency characteristics of these nine different stages, due to patent concerns and as Miracurall is publicly not available yet in the world market. At the rear side, there are two socket points, one is for the connecting cord and the other is for external adaptor supply. There are also two battery caps for connecting nine volts batteries. The connecting cord has two alligator clips at one end that can be attached to two small metal plates. These metal plates get placed in between layers of wet cotton cloth wrapped on the wrists. Once the entire circuit is completed, the administration can begin in an exact time pattern. After each administration, consumption of a glass of drinking water at room temperature flushes out the non-lethal toxins that are produced inside the body due to the interaction between the M-Pulses and the anatomy. Usually the daily administration period varies between thirty to sixty minutes. Over time classic traits of both Type-1 and Type-2 DM like polyuria, polydipsia, weight loss, blurred vision, polyphagia, generalized weakness, diabetic ketoacidosis and diabetic neuropathy start to disappear as bodily homeostasis gets reinstated. For Type-1 DM, over time the individuals need to keep reducing the amount of their insulin needs until it reaches at zero need for insulin consumption. The total recovery period varies between individuals depending upon their physical conditions. To achieve the completely cured state, it may take from four to fourteen months. Once cured, the health of the individual lies in his/her own hand. The individual can live healthily ever after if he/she keeps following a healthy lifestyle with a healthy diet. The volunteers, on whom I ran my primary trial for many months, are now free from Hyperglycemia and are leading their lives without the need for any medication or insulin consumption. Presently I'm about to begin a bigger trial of Miracurall worldwide, before it gets available in the world market for the general public. For this trial process, I gladly welcome distinguished scientists and doctors from all around the planet to join hands together in the betterment of life on earth.

\section{Summary}

Diabetes Mellitus is just the beginning of this miraculous journey. By curing Hyperglycemia, it is just exemplified that where there is a disease, there is a cure for that disease too. Further study and research will reveal more profound details and illustrations on the curative properties of Miracurall and will shed an inexplicable ray of hope on other diseases that torment life on planet earth. We just need to break our own barriers of thoughts and conventions, and look for the quite evident cure in some unimaginable arenas of science. Science is never stagnant, it is always advancing forward, leaving behind the signatures of those advancements which we have made just a while ago. There is a lot to explore, a lot to learn, a lot to see with the eyes of our boundless mind that never gets exhausted by asking questions beyond the present plausibility. It is the privilege of a researcher to find out the answers to those questions that have been tormenting mankind for centuries. There is no other pleasure in the humanly imaginable cosmos, that can be the same as the pleasure of finding out how do mother nature and everything in its womb work. 


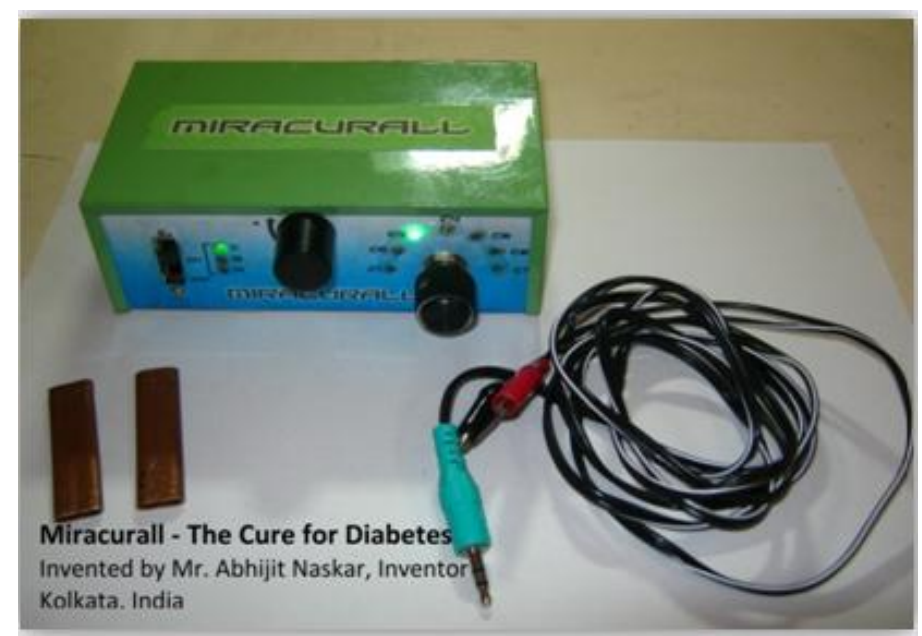

\section{References}

[1]. Denovan P. Begg and Stephen C. Woods, Interactions between the central nervous system and pancreatic islet secretions: a historical perspective, 2013

[2]. Richard Alan Miller and Iona Miller, The Schumann's Resonance and Human Physiobiology, 2003

[3]. Kristl Vonck and Paul Boon, The Mechanism of Action of Vagus Nerve Stimulation Therapy, 2008

[4]. Taber's Cyclopedic Medical Dictionary 21

[5]. Andrew Tresidder, Vibrational Medicine, Allopathic Medicine, Flower Essence Use and Paradigms and Challenges in Healthcare, 2013

[6]. James L. Oschman, Energy Medicine - The Scientific Basis, 2000

[7]. Richard S. Snell, Clinical Neuroanatomy, 2010

[8]. Universe : The Definitive Visual Guide, 2012 